\title{
Hyperspaces of Superparacompact Spaces and Continuous Maps
}

\author{
Adilbek Ataxanovich Zaitov ${ }^{1 *}$ and Davron Ilxomovich Jumaev ${ }^{1}$ \\ ${ }^{1 *}$ Tashkent institute of architecture and civil engineering, Tashkent, Uzbekistan \\ * Corresponding author
}

\section{Article Info}

Keywords: Finite-component cover, Hyperspace, Superparacompact space, Tychonoff map

2010 AMS: 54B20, 54C10.

Received: 9 November 2018

Accepted: 9 April 2019

Available online: 28 June 2019

\begin{abstract}
In the present paper we establish that the $\operatorname{space} \exp _{\beta} X$ of compact subsets of a Tychonoff space $X$ is superparacompact iff $X$ is so. Further, we prove the Tychonoff map $\exp _{\beta} f$ : $\exp _{\beta} X \rightarrow \exp _{\beta} Y$ is superparacompact iff a given map $f: X \rightarrow Y$ is superparacompact.
\end{abstract}

\section{Introduction}

In the present paper under space we mean a topological $T_{1}$-space, under compact a Hausdorff compact space and under map a continuous map.

A collection $\omega$ of subsets of a set $X$ is said [1] to be star-countable (respectively, star-finite) if each element of $\omega$ intersects at most a countable (respectively, finite) set of elements of $\omega$. A collection $\omega$ of subsets of a set $X$ refines a collection $\Omega$ of subsets of $X$ if for each element $A \in \omega$ there is an element $B \in \Omega$ such that $A \subset B$. They also say that $\omega$ is a refinement of $\Omega$.

A finite sequence of subsets $M_{0}, \ldots, M_{s}$ of a set $X$ is [2] a chain connecting sets $M_{0}$ and $M_{s}$, if $M_{i-1} \cap M_{i} \neq \varnothing$ for $i=1, \ldots, s$. A collection $\omega$ of subsets of a set $X$ is said to be connected if for any pair of sets $M, M^{\prime} \subset X$ there exists a chain in $\omega$ connecting sets $M$ and $M^{\prime}$. The maximal connected subcollections of $\omega$ are called components of $\omega$. A star-finite open cover of a space $X$ is said to be $a$ finite-component cover if the number of elements of each component is finite. A space $X$ is said to be superparacompact if every open cover of $X$ has a finite-component cover which refines it.

Note that any compact space is superparacompact, and any superparacompact space is strongly paracompact. Infinite discrete space is superparacompact, but it is not compact. Real line is strongly paracompact, but it is not superparacompact.

For a collection $\omega=\left\{O_{\alpha}: \alpha \in A\right\}$ of subsets of a space $X$ we suppose $[\omega]=[\omega]_{X}=\left\{\left[O_{\alpha}\right]_{X}: \alpha \in A\right\}$. For a space $X$, its some subspace $W$ and a set $B \subset X \backslash W$ they say [2] that an open cover $\lambda$ of the space $W$ pricks out the set $B$ in $X$ if $B \cap\left(\cup[\lambda]_{X}\right)=\varnothing$.

The following criterion plays a key role in investigation the class of superparacompact spaces.

Theorem 1.1. [3] A Tychonoff space $X$ is superparacompact iff for every closed set $F$ in $\beta X$ lying in the growth $\beta X \backslash X$ there exists a finite-component cover $\lambda$ of $X$ pricking out $F$ in $\beta X\left(i . e . F \cap\left(\cup[\lambda]_{\beta X}\right)=\varnothing\right)$.

D.Buhagiar and T.Miwa offered the following criterion of superparacompactness.

Theorem 1.2. [4] A Tychonoff space $X$ is superparacompact iff for every closed set $F$ in perfect compactification bX lying in the growth $b X \backslash X$ there is a finite-component cover $\lambda$ of $X$ pricking out $F$ in $b X$ (i. e. $\left.F \cap\left(\cup[\lambda]_{b X}\right)=\varnothing\right)$.

Let us recall a notion of the perfect compactification. For a topological space $X$ and its subset $A$ a set $F r_{X} A=[A]_{X} \cap[X \backslash A]_{X}=[A]_{X} \backslash \operatorname{Int} t_{X} A$ is called [5] a boundary of $A$.

Let $v X$ be a compact extension of a Tychonoff space $X$. If $H \subset X$ is an open set in $X$, then by $O(H)$ (or by $O_{v X}(H)$ ) we denote a maximal open set in $v X$ satisfying $O_{v X}(H) \cap X=H$. It is easy to see that

$$
O_{v X}(H)=\bigcup_{\substack{\Gamma \in \tau_{v X}, \Gamma \cap X=H}} \Gamma,
$$


where $\tau_{v X}$ is the topology of the space $v X$.

A compactification $v X$ of a Tychonoff space $X$ is called perfect with respect to an open set $H$ in $X$, if the equality $\left[F r_{X} H\right]_{v X}=F r_{v X} O_{v X}(X)$ holds. If $v X$ is perfect with respect to every open set in $X$, then it is called a perfect compactification of the space $X$ ([1], P. 232). A compactification $v X$ of a space $X$ is perfect iff for any two disjoint open sets $U_{1}$ and $U_{2}$ in $X$ the equality $O\left(U_{1} \cup U_{2}\right)=O\left(U_{1}\right) \cup O\left(U_{2}\right)$ is carried out. The Stone-Cěch compactification $\beta X$ of $X$ is perfect. The equality $O\left(U_{1} \cup U_{2}\right)=O\left(U_{1}\right) \cup O\left(U_{2}\right)$ is satisfied for every pair of open sets $U_{1}$ and $U_{2}$ in $X$ iff $X$ is normal, and the compactification $v X$ coincides with the Stone-Cěch compactification $\beta X$, i. e. $v X \cong \beta X$. Let $X$ be a space. By $\exp X$ we denote a set of all nonempty closed subsets of $X$. A family of sets of the view

$$
O\left\langle U_{1}, \ldots, U_{n}\right\rangle=\left\{F \in \exp X: F \subset \bigcup_{i=1}^{n} U_{n}, F \cap U_{1} \neq \varnothing, \ldots, F \cap U_{n} \neq \varnothing\right\}
$$

forms a base of a topology on $\exp X$, where $U_{1}, \ldots, U_{n}$ are open nonempty sets in $X$. This topology is called the Vietoris topology. A space $\exp X$ equipped with Vietoris topology is called hyperspace of $X$. For a compact space $X$ its hyperspace $\exp X$ is also a compact space (for details, see [6], [7], [8]).

Note for any space $X$ it is well known that

$$
\left[O\left\langle U_{1}, \ldots, U_{n}\right\rangle\right]_{\exp X}=O\left\langle\left[U_{1}\right]_{X}, \ldots,\left[U_{n}\right]_{X}\right\rangle .
$$

Let $f: X \rightarrow Y$ be continuous map of compacts, $F \in \exp X$. We put

$$
(\exp f)(F)=f(F) \text {. }
$$

This equality defines a map $\exp f: \exp X \rightarrow \exp Y$. For a continuous map $f$ the map $\exp f$ is continuous. Really, it follows from the formula

$$
(\exp f)^{-1} O\left\langle U_{1}, \ldots, U_{m}\right\rangle=O\left\langle f^{-1}\left(U_{1}\right), \ldots, f^{-1}\left(U_{m}\right)\right\rangle
$$

what one can check directly. Note that if $f: X \rightarrow Y$ is an epimorphism, then $\exp f$ is also an epimorphism.

For a Tychonoff space $X$ we put

$$
\exp _{\beta} X=\{F \in \exp \beta X: F \subset X\} .
$$

It is clear, that $\exp _{\beta} X \subset \exp X$. Consider the set $\exp _{\beta} X$ as a subspace of the space $\exp X$. For a Tychonoff $\operatorname{spaces} X$ the $\operatorname{space} \exp _{\beta} X$ is also a Tychonoff space with respect to the induced topology.

For a continuous map $f: X \rightarrow Y$ of Tychonoff spaces we put

$$
\exp _{\beta} f=\left.(\exp \beta f)\right|_{\exp _{\beta} X},
$$

where $\beta f: \beta X \rightarrow \beta Y$ is the Stone-Cěch compactification [5] of $f$ (it is unique).

As it is well-known the action of functors on various categories of topological spaces and their continuous maps is one of the main problems of theory of covariant functors, in the present paper we investigate the action of the functor exp (the construction of taking of a hyperspace of a given space) on superparacompact spaces (section 2) and superparacompact maps (section 3).

\section{Hyperspace of superparacompact spaces}

It is well known that for a Tychonoff space $X$ the set $\exp _{\beta} X$ is everywhere dense in $\exp \beta X$, i. e. $\exp \beta X$ is a compactification of the space $\exp _{\beta} X$. We claim $\exp \beta X$ is a perfect compactification of $\exp _{\beta} X$. At first we will prove the following technical statement.

Lemma 2.1. Let $\gamma X$ be a compact extension of a space $X$ and, $V$ and $W$ be disjoint open sets in $\gamma X$. Let $V^{X}=X \cap V$ and $W^{X}=X \cap W$. Then the following equality is true:

$$
\left[X \backslash V^{X}\right]_{\gamma X} \cap\left[X \backslash W^{X}\right]_{\gamma X}=\left[X \backslash\left(V^{X} \cup W^{X}\right)\right]_{\gamma X} .
$$

Proof. It is clear that $\left[X \backslash V^{X}\right]_{\gamma X} \cap\left[X \backslash W^{X}\right]_{\gamma X} \supset\left[X \backslash\left(V^{X} \cup W^{X}\right)\right]_{\gamma X}$. Let $x \in\left[X \backslash V^{X}\right]_{\gamma X} \cap\left[X \backslash W^{X}\right]_{\gamma X}$. Then each open neighbourhood $O x$ in $\gamma X$ of $x$ intersects with the sets $X \backslash V^{X}$ and $X \backslash W^{X}$. Hence, $O x \not \subset V^{X}$ and $O x \not \subset W^{X}$. Therefore, since $V^{X} \cap W^{X}=\varnothing$, we have $O x \not \subset V^{X} \cup W^{X}$, i. e. $O x \cap X \backslash\left(V^{X} \cup W^{X}\right) \neq \varnothing$. By virtue of arbitrariness of the neighbourhood $O x$ we conclude that $x \in\left[X \backslash\left(V^{X} \cup W^{X}\right)\right]_{\gamma X}$.

Theorem 2.2. For a Tychonoff space $X$ the space $\exp \beta X$ is a perfect compactification of the space $\exp _{\beta} X$.

Proof. It is enough to consider basic open sets. Let $U_{1}$ and $U_{2}$ be disjoint open sets in $X$. Since $\beta X$ is perfect compactification of $X$ we have $O_{\beta X}\left(U_{1} \cup U_{2}\right)=O_{\beta X}\left(U_{1}\right) \cup O_{\beta X}\left(U_{2}\right)$. Consider open sets

$$
O\left\langle U_{i}\right\rangle=\left\{F: F \in \exp _{\beta} X, F \subset U_{i}\right\}, \quad i=1,2
$$

in $\exp _{\beta} X$. It is clear, that $O\left\langle U_{1}\right\rangle \cap O\left\langle U_{2}\right\rangle=\varnothing$. We will show that

$$
O_{\exp \beta X}\left(O\left\langle U_{1}\right\rangle \cup O\left\langle U_{2}\right\rangle\right)=O_{\exp \beta X}\left(O\left\langle U_{1}\right\rangle\right) \cup O_{\exp \beta X}\left(O\left\langle U_{2}\right\rangle\right) .
$$

The inclusion $\supset$ follows from the definition of the set $O(H)$ (see [1], P. 234). That is why it is enough to show the inverse inclusion. Let $\Phi \subset \beta X$ be a closed set such that $\Phi \notin O_{\exp \beta X}\left(O\left\langle U_{1}\right\rangle\right) \cup O_{\exp \beta X}\left(O\left\langle U_{2}\right\rangle\right)$. Then $\Phi \in \exp \beta X \backslash O_{\exp \beta X}\left(O\left\langle U_{i}\right\rangle\right), i=1,2$. From [1] (see, P. 234) we have

$$
\exp \beta X \backslash O_{\exp \beta X}\left(O\left\langle U_{i}\right\rangle\right)=\left[\exp _{\beta} X \backslash O\left\langle U_{i}\right\rangle\right]_{\exp \beta X}, \quad i=1,2 .
$$


Hence $\Phi \in\left[\exp _{\beta} X \backslash O\left\langle U_{i}\right\rangle\right]_{\exp \beta X}, i=1,2$. Since $O\left\langle U_{1}\right\rangle \cap O\left\langle U_{2}\right\rangle=\varnothing$ by Lemma 2.1 we have

$$
\left[\exp _{\beta} X \backslash O\left\langle U_{1}\right\rangle\right]_{\exp \beta X} \cap\left[\exp _{\beta} X \backslash O\left\langle U_{2}\right\rangle\right]_{\exp \beta X}=\left[\exp _{\beta} X \backslash O\left(\left\langle U_{1}\right\rangle \cup O\left\langle U_{2}\right\rangle\right)\right]_{\exp \beta X} .
$$

Therefore, $\Phi \in\left[\exp _{\beta} X \backslash O_{\exp \beta X}\left(O\left\langle U_{1}\right\rangle \cup O\left\langle U_{2}\right\rangle\right)\right]_{\exp \beta X}$, what is equivalent $\Phi \in \exp \beta X \backslash O_{\exp \beta X}\left(\left\langle U_{1}\right\rangle \cup\left\langle U_{2}\right\rangle\right)$ (see [1], P. 234). In other words, $\Phi \notin O_{\exp \beta X}\left(\left\langle U_{1}\right\rangle \cup\left\langle U_{2}\right\rangle\right)$. Thus, we have established that inclusion $O_{\exp \beta X}\left(\left\langle U_{1}\right\rangle \cup\left\langle U_{2}\right\rangle\right) \subset O_{\exp \beta X}\left(O\left\langle U_{1}\right\rangle\right) \cup O_{\exp \beta X}\left(O\left\langle U_{2}\right\rangle\right)$ is also fair.

Lemma 2.3. Let $U_{1}, \ldots, U_{n} ; V_{1}, \ldots, V_{m}$ be open subsets of a space $X$. Then $O\left\langle U_{1}, \ldots, U_{n}\right\rangle \cap O\left\langle V_{1}, \ldots, V_{m}\right\rangle \neq \varnothing$ iff for each $i \in\{1, \ldots, n\}$ and for each $j \in\{1, \ldots, m\}$ there exists, respectively $j(i) \in\{1, \ldots, m\}$ and $i(j) \in\{1, \ldots, n\}$, such that $U_{i} \cap V_{j(i)} \neq \varnothing$ and $U_{i(j)} \cap V_{j} \neq \varnothing$.

Proof. Assume that for every $i \in\{1, \ldots, n\}$ there exists $j(i) \in\{1, \ldots, m\}$ such that $U_{i} \cap V_{j(i)} \neq \varnothing$ and for every $j \in\{1, \ldots, m\}$ there exists $i(j) \in\{1, \ldots, n\}$ such that $U_{i(j)} \cap V_{j} \neq \varnothing$. For any pair $(i, j) \in\{1, \ldots, n\} \times\{1, \ldots, m\}$ for which $U_{i} \cap V_{j} \neq \varnothing$, choose a point $x_{i j} \in U_{i} \cap V_{j}$ and make a closed set $F$ consisting of these points. Then $F \subset \bigcup_{i=1}^{n} U_{i}$ and $F \subset \bigcup_{j=1}^{m} V_{j}$. Besides, $F \cap U_{i} \neq \varnothing, i=1, \ldots, n$, and $F \cap V_{j} \neq \varnothing$, $j=1, \ldots, m$. Therefore, $F \in O\left\langle U_{1}, \ldots, U_{n}\right\rangle \cap O\left\langle V_{1}, \ldots, V_{m}\right\rangle$.

Suppose there exists $i_{0} \in\{1, \ldots, n\}$ such that $U_{i_{0}} \cap V_{j}=\varnothing$ for all $j \in\{1, \ldots, m\}$. Then $U_{i_{0}} \cap \bigcup_{j=1}^{m} V_{j}=\varnothing$ and for each $F \in O\left\langle U_{1}, \ldots, U_{n}\right\rangle$ we have $F \not \subset \bigcup_{j=1}^{m} V_{j}$. Hence, $F \notin O\left\langle V_{1}, \ldots, V_{m}\right\rangle$. Similarly, every $\Gamma \in O\left\langle V_{1}, \ldots, V_{m}\right\rangle$ lies in $\bigcup_{j=1}^{m} V_{j}$ what implies $\Gamma \cap U_{i_{0}}=\varnothing$. From here $\Gamma \notin O\left\langle U_{1}, \ldots, U_{n}\right\rangle$. Thus, $O\left\langle U_{1}, \ldots, U_{n}\right\rangle \cap O\left\langle V_{1}, \ldots, V_{m}\right\rangle=\varnothing$.

Lemma 2.4. Let $v$ be a finite-component cover of a Tychonoff space $X$. Then the family $\exp _{\beta} v=\left\{O\left\langle U_{1}, \ldots, U_{n}\right\rangle: U_{i} \in v, i=1, \ldots, n ; n \in\right.$ $\mathbb{N}\}$ is a finite-component cover of the space $\exp _{\beta} X$.

Proof. Let $O\left\langle G_{1}, \ldots, G_{k}\right\rangle$ be an element of $\exp _{\beta} v$. Each $G_{i} \in v$ intersects with finite elements of $v$. Let $\left|\left\{\alpha: G_{i} \cap U_{\alpha} \neq \varnothing, U_{\alpha} \in v\right\}\right|=n_{i}$, $i=1,2, \ldots, k$. Denote $\gamma=\left\{G_{i} \cap U_{j}: G_{i} \cap U_{j} \neq \varnothing, i=1,2, \ldots, k, U_{j} \in v\right\}$. Then $|\gamma| \leq n_{1} \cdot \ldots \cdot n_{k}$. Therefore, the set $O\left\langle G_{1}, \ldots, G_{k}\right\rangle$ crosses not more then $\prod_{i=1}^{k} n_{i}$ elements of $\exp _{\beta} v$. It means that the collection $\exp _{\beta} v$ is star-finite.

Let $F \in \exp _{\beta} X$. There is a subfamily $v_{F} \subset v$ such that $F \subset \bigcup_{U \in v_{F}} U$. From a cover $\left\{F \cap U: U \in v_{F}, F \cap U \neq \varnothing\right\}$ of the compact $F$ it is possible to allocate a finite subcover $\left\{F \cap U_{i}: i=1, \ldots, m\right\}$. We have $F \in O\left\langle U_{1}, \ldots, U_{m}\right\rangle$. So, the family $\exp _{\beta} v$ is a $\operatorname{cover}$ of $\exp _{\beta} X$. On the other hand by the definition of Vietoris topology the cover $\exp _{\beta} v$ is open. Thus, $\exp _{\beta} v$ is a star-finite open $\operatorname{cover}$ of $\exp _{\beta} X$.

We will show now that all components of the $\exp _{\beta} v$ are finite.

Let $M=O\left\langle G_{1}, \ldots, G_{s}\right\rangle$ and $M^{\prime}=O\left\langle G_{1}^{\prime}, \ldots, G_{t}^{\prime}\right\rangle$ be arbitrary elements of $\exp _{\beta} v$. Further, let $\gamma_{G_{i} G_{j}^{\prime}}=\left\{U_{l}^{i j}: l=1,2, \ldots, n_{i j}\right\}$ be the maximal chain of $v$ connecting $G_{i}$ and $G_{j}^{\prime}, i=1,2, \ldots, s ; j=1,2, \ldots, t$. By definition these sets satisfy the following properties:

(1) $U_{1}^{i j}=G_{i}, \quad i=1, \ldots, s ; j=1, \ldots, t$;

(2) $U_{n_{i j}}^{i j}=G_{j}^{\prime}, \quad i=1, \ldots, s ; j=1, \ldots, t$;

(3) $U_{l}^{i j} \cap U_{l+1}^{i j} \neq \varnothing, \quad l=1, \ldots, n_{i j}-1 ; i=1, \ldots, s ; j=1, \ldots, t$.

If $s<t$ we have $O\left\langle G_{1}, \ldots, G_{s}\right\rangle=O\left\langle U_{1}^{1 j}, \ldots, U_{1}^{s j}, U_{1}^{i_{1}(s+1)}, \ldots, U_{1}^{i_{t-s} t}\right\rangle$, where $j=1, \ldots, t$ and $i_{1}, \ldots, i_{t-s} \in\{1, \ldots, s\}$. Further, $O\left\langle G_{1}^{\prime}, \ldots, G_{t}^{\prime}\right\rangle=$ $O\left\langle U_{n_{i 1}}^{i 1}, \ldots, U_{n_{i t}}^{i t}\right\rangle, i=1, \ldots, s$. Thus, the $\operatorname{cover} \exp _{\beta} v$ has a chain connecting the given sets $M=O\left\langle G_{1}, \ldots, G_{s}\right\rangle$ and $M^{\prime}=O\left\langle G_{1}^{\prime}, \ldots, G_{t}^{\prime}\right\rangle$. The case $s>t$ is analogously.

Now using Lemma 2.1 and calculating directly we find that each maximal chain of $\exp _{\beta} v$ connecting the sets $M=O\left\langle G_{1}, \ldots, G_{s}\right\rangle$ and $M^{\prime}=O\left\langle G_{1}^{\prime}, \ldots, G_{t}^{\prime}\right\rangle$ has no more than $\prod_{\substack{i=1, j=1}}^{t} n_{i j}$ elements. Thus, all components of $\exp _{\beta} v$ is finite.

Theorem 2.5. For a Tychonoff space $X$ its hyperspace $\exp _{\beta} X$ is superparacompact iff $X$ is superparacompact.

Proof. As the superparacompactness is inherited to the closed subsets [2], the superparacompactness of $\exp _{\beta} X$ implies superparacompactness of the closed subset $X \subset \exp _{\beta} X$.

Let $\Omega$ be an open cover of $\exp _{\beta} X$. For each element $G \in \Omega$ there exists $O_{G}\left\langle U_{1}, \ldots, U_{n}\right\rangle$ such that $O_{G}\left\langle U_{1}, \ldots, U_{n}\right\rangle \subset G$, where $U_{1}, \ldots, U_{n}$ are open sets in $X$. We can choose sets $G \in \Omega$ so that a collection of sets $O_{G}\left\langle U_{1}, \ldots, U_{n}\right\rangle$ forms a cover of $\exp _{\beta} X$, what we denote by $\Omega^{\prime}$. It is easy to see that a collection $\omega^{\prime}=\underset{O_{G}\left\langle U_{1}, \ldots, U_{n}\right\rangle \in \Omega^{\prime}}{\bigcup}\left\{U_{1}, \ldots, U_{n}\right\}$ is an open cover of $X$. There exists a finite-component cover $\omega$ of $X$ which refines $\omega^{\prime}$. Then by Lemma 2.4 the collection

$$
\exp _{\beta} \omega=\left\{O\left\langle V_{1}, \ldots, V_{k}\right\rangle: V_{i} \in \omega, i=1, \ldots, n ; n \in \mathbb{N}\right\}
$$

is a finite-component cover of $\exp _{\beta} X$ and it refines $\Omega$. 


\section{Superparacompactness of the $\operatorname{map} \exp _{\beta} f$}

For a continuous map $f:\left(X, \tau_{X}\right) \rightarrow\left(Y, \tau_{Y}\right)$ and $O \in \tau_{Y}$ a preimage $f^{-1} O$ is called $a$ tube (above $O$ ). Remind, a continuous map $f: X \rightarrow Y$ is called [2] a $T_{0}$-map, if for each pair of distinct points $x, x^{\prime} \in X$, such that $f(x)=f\left(x^{\prime}\right)$, at least one of these points has an open neighbourhood in $X$ which does not contain another point. A continuous map $f: X \rightarrow Y$ is called totally regular, if for each point $x \in X$ and every closed set $F$ in $X$ not containing $x$ there exists an open neighbourhood $O$ of $f(x)$ such that in the tube $f^{-1} O$ the sets $\{x\}$ and $F$ are functional separable. Totally regular $T_{0}$-map is said to be a Tychonoff map.

Obviously, each continuous map $f: X \rightarrow Y$ of a Tychonoff space $X$ into a topological space $Y$ is a Tychonoff map. In this case owing to the set $\exp _{\beta} X$ is a Tychonoff space concerning to Vietoris topology for every Tychonoff space $X$, the map $\exp _{\beta} f: \exp _{\beta} X \rightarrow \exp _{\beta} Y$ is a Tychonoff map.

A continuous, closed map $f: X \rightarrow Y$ is said to be compact if the preimage $f^{-1} y$ of each point $y \in Y$ is compact. A continuous map $f: X \rightarrow Y$ is compact iff for each point $y \in Y$ and every cover $\omega$ of the fibre $f^{-1} y$, consisting of open sets in $X$, there is an open neighbourhood $O$ of $y$ in $Y$ such that the tube $f^{-1} O$ can be covered with a finite subfamily of $\omega$.

A compact map $b f: b_{f} X \rightarrow Y$ is said to be a compactification of a continuous map $f: X \rightarrow Y$ if $X$ is everywhere dense in $b_{f} X$ and $\left.b f\right|_{X}=f$. On the set of all compactifications of the map $f$ it is possible to introduce a partial order: for the compactifications $b_{1} f: b_{1 f} X \rightarrow Y$ and $b_{2} f: b_{2 f} X \rightarrow Y$ of $f$ we put $b_{1} f \leq b_{2} f$ if there is a natural map of $b_{2 f} X$ onto $b_{1 f} X$. B. A. Pasynkov showed that for each Tychonoff map $f: X \rightarrow Y$ there exists its maximal compactification $g: Z \rightarrow Y$, which he denoted by $\beta f$, and the space $Z$ where this maximal compactification defines by $\beta_{f} X$. To within homeomorphism for a given Tychonoff map $f$ its maximal compactification $\beta f$ is unique.

Remark 3.1. Note that the maps $b_{1} f, b_{2} f, \beta f$ are compactifications of the map $f$. The spaces $b_{1 f} X, b_{2 f}, \beta_{f} X$ are some extensions of $X$ but they are not obliged to be compactifications.

A Tychonoff map $f: X \rightarrow Y$ is said to be superparacompact, if for every closed set $F$ in $\beta_{f} X$ lying in the growth $\beta_{f} X \backslash X$ there exists a finite-component cover $\lambda$ of $X$ pricking out $F$ in $\beta_{f} X$ (i. e. $F \cap\left(\cup[\lambda]_{\beta_{f} X}\right)=\varnothing$ ) [3].

It is easy to see that one can define superparacompactness of a map as follows: a map $f: X \rightarrow Y$ is superparacompact if for each $y \in Y$ and every open cover $\Upsilon$ of $f^{-1} y$ in $X$ there exists an open neighbourhood $O$ of $y$ in $Y$ such that $\Upsilon$ has a finite-component cover $v$ of $f^{-1} O$ in $X$ which refines $\Upsilon$.

Definition 3.2. A compactification $b f: b_{f} X \rightarrow Y$ of a Tychonoff map $f: X \rightarrow Y$ is said to be perfect compactification of $f$ if for each point $y \in Y$ and for every disjoint open sets $U_{1}$ and $U_{2}$ in $X$ there exists an open neighbourhood $O \subset Y$ of $y$ such that the equality

$$
O_{b_{f} X}\left(U_{1} \cup U_{2}\right) \cap b f^{-1} O=\left(O_{b_{f} X}\left(U_{1}\right) \cup O_{b_{f} X}\left(U_{2}\right)\right) \cap b f^{-1} O
$$

holds.

Let $f: X \rightarrow Y$ be a continuous map of a Tychonoff space $X$ into a space $Y$. It is well known there exists a compactification $v X$ of $X$ such that $f$ has a continuous extension $v f: v X \rightarrow Y$ on $v X$. It is clear, $v f$ is a perfect compactification of $f$.

The following result is an analog of Theorem 1.2 for a case of maps.

Theorem 3.3. Let $b f: b_{f} X \rightarrow Y$ be a perfect compactification of a Tychonoff map $f: X \rightarrow Y$. The map $f$ is superparacompact iff for every closed set $F$ in $b_{f} X$ lying in the growth $b_{f} X \backslash X$ there exists a finite-component cover $\lambda$ of $X$ pricking out the set $F$ in $b_{f} X$.

Proof. The proof is carried out similar to the proof of Theorem $1.1 \Pi$ from [2].

Evidently a restriction $\left.f\right|_{\Phi}: \Phi \rightarrow Y$ of a superparacompact map $f: X \rightarrow Y$ on the closed subset $\Phi \subset X$ is a superparacompact map. The following result is a variant of Theorem 2.2 for a case of maps.

Theorem 3.4. Let $f: X \rightarrow Y$ be a Tychonoff map. Then the map $\exp _{\beta} \beta f: \exp _{\beta} \beta_{f} X \rightarrow \exp _{\beta} Y$ is a perfect compactification of $\exp _{\beta} f:$ $\exp _{\beta} X \rightarrow \exp _{\beta} Y$.

Proof. The proof is similar to the proof of Theorem 2.2. Here the equality

$$
\left(\exp _{\beta} \beta f\right)^{-1} O\left\langle U_{1}, \ldots, U_{m}\right\rangle=O\left\langle\beta f^{-1}\left(U_{1}\right), \ldots, \beta f^{-1}\left(U_{m}\right)\right\rangle
$$

is used.

The following statement is the main result of this section.

Theorem 3.5. The Tychonoff map $\exp _{\beta} f: \exp _{\beta} X \rightarrow \exp _{\beta} Y$ is superparacompact iff a map $f: X \rightarrow Y$ is superparacompact.

Proof. Let $\exp _{\beta} f: \exp _{\beta} X \rightarrow \exp _{\beta} Y$ be a superparacompact map. It implies that $f: X \rightarrow Y$ is a superparacompact map since $X \cong \exp _{1} X$ is closed set in $\exp _{\beta} X$.

Let now $f: X \rightarrow Y$ be a superparacompact map. Consider arbitrary $\Gamma \in \exp _{\beta} Y$ and an open cover $\Omega$ of $\left(\exp _{\beta} f\right)^{-1}(\Gamma)=\left\{F \in \exp _{\beta} X\right.$ : $f(F)=\Gamma\}$ in $\exp _{\beta} X$. For each element $G \in \Omega$ there exists $O_{G}\left\langle U_{1}, \ldots, U_{n}\right\rangle$ such that $O_{G}\left\langle U_{1}, \ldots, U_{n}\right\rangle \subset G$, where $U_{1}, \ldots, U_{n}$ are open sets in $X$. We can choose sets $G \in \Omega$ so that a collection of sets $O_{G}\left\langle U_{1}, \ldots, U_{n}\right\rangle$ forms a cover of $\left(\exp _{\beta} f\right)^{-1}(\Gamma)$, what we denote by $\Omega^{\prime}$. It is easy to see that a collection $\omega^{\prime}=\bigcup_{O_{G}\left\langle U_{1}, \ldots, U_{n}\right\rangle \in \Omega^{\prime}}\left\{U_{1}, \ldots, U_{n}\right\}$ is an open cover of $f^{-1} \Gamma$ in $X$. For each $y \in \Gamma$ there exists an open neighbourhood $O_{y}$ of $y$ in $Y$ such that the collection $\omega_{y}=\left\{U \cap f^{-1} O_{y}: U \in \omega^{\prime}\right\}$ is an open cover of $f^{-1} y$ in $X$ and $\omega_{y}$ has a finite-component cover $\omega_{y}^{\prime}$ of $f^{-1} O_{y}$ in $X$ which refines $\omega_{y}$. Gather such $O_{y}$ and construct an open cover $\left\{O_{y}: y \in \Gamma\right\}$ of $\Gamma$ in $Y$. Since $\Gamma \in \exp _{\beta} Y$ by construction of hyperspace, $\Gamma$ is a compact subset of $Y$. Consequently, there exists a finite open subcover $\gamma=\left\{O_{y_{1}}, \ldots, O_{y_{n}}\right\}$ in $Y$, which covers $\Gamma$. Put 
$\omega=\bigcup_{y_{i} \in \gamma} \omega_{y_{i}}^{\prime}$. Then $\omega$ is an open cover of $f^{-1}\left(\bigcup_{U \in \omega} U\right)$ in $X$. By the construction $\omega$ is a finite-component cover, and it refines $\omega^{\prime}$. Hence, $\exp _{\beta} \omega$ is a finite-component cover of $\left(\exp _{\beta} f\right)^{-1} O\left\langle O_{y_{1}}, \ldots, O_{y_{n}}\right\rangle=\left\langle f^{-1} O_{y_{1}}, \ldots, f^{-1} O_{y_{n}}\right\rangle$ in $\exp _{\beta} X$ and it refines $\Omega$.

So, for each $\Gamma \in \exp _{\beta} Y$ and every open cover $\Omega$ of $\left(\exp _{\beta} f\right)^{-1} \Gamma$ in $\exp _{\beta} X$ there exists an open neighbourhood $O\left\langle O_{y_{1}}, \ldots, O_{y_{n}}\right\rangle$ of $\Gamma$ in $\exp _{\beta} Y$ such that $\Omega$ has a finite-component cover $\exp _{\beta} \omega$ of $\left(\exp _{\beta} f\right)^{-1} O\left\langle O_{y_{1}}, \ldots, O_{y_{n}}\right\rangle$ in $\exp _{\beta} X$ which refines $\Omega$. Thus, the map $\exp _{\beta} f: \exp _{\beta} X \rightarrow \exp _{\beta} Y$ is superparacompact.

Corollary 3.6. Let $f: X \rightarrow Y$ be a superparacompact map and $\Phi$ be a closed set in $\exp _{\beta} \beta_{f} X$ such that $\Phi \subset \exp _{\beta} \beta_{f} X \backslash \exp _{\beta} X$. Then

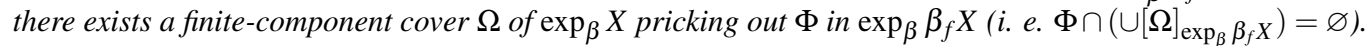

Corollary 3.7. The functor $\exp _{\beta}$ lifts onto category of superparacompact spaces and their continuous maps.

\section{Acknowledgments}

The authors would like to acknowledge the comprehensive backing of professor Sh.Ayupov. Also the authors would like to express gratitude to the reviewers and Prof. Dr. Fuat Usta for the revealed shortcomings, the specified remarks, corrections and useful advices.

\section{References}

[1] A. V. Arkhangelsky, V. I. Ponomarev. Fundamentals of the General Topology: Problems and Exercises. - D.Reidel Publishing Company. 1983. - 415 pp (Originally published as: Osnovy Obsheii Topologii v Zadachakh i Uprajneniyakh, by A. V. Arkhangelsky, V. I. Ponomarev, Izdatel'stvo 'Nauka' Moscow, 1974).

[2] D. K. Musayev, B. A. Pasynkov, On compactness and completeness properties of topological spaces and continuous maps, - Tashkent: 'Fan'. 1994. $124 \mathrm{pp}$.

[3] D. K. Musayev, On compactness and completeness properties of topological spaces and continuous maps, - Tashkent: 'NisoPoligraf'. 2011. - 216 pp.

[4] D. Buhagiar, T. Miwa, On Superparacompact and Lindelof GO-Spaces, Houston J. Math., (24)3, (1998), 443 - 457.

[5] R. Engelking, General Topology, - Polish Scientific Publishers. Warszawa. - 1977.

[6] V. V. Fedorchuk, V. V. Filippov, General Topology. Basic Constructions (in Russian). - Moscow. Fizmatlit. 2006.

[7] R. Bartsch, Hyperspaces in topological categories, //arXiv:1410.3137v2 [math.GN] September 4, 2018. P. 13.

[8] V. Gutev, Hausdorff continuous sections, J. Math. Soc. Japan., (66) 2, (2014), pp. 523-534. doi: 10.2969/jmsj/06620523 